\title{
Zweiter Kommentar zu den Kosten unseres Gesundheitswesens
}

Jean-Pierre Pochon

Korrespondenz:

PD Dr. med. Jean-Pierre Pochon Tagesklinik für Kinderchirurgie Dübendorfstrasse 20

CH-8117 Fällanden

pochon@ambikid.ch
Lieber Herr Dr. Kocher, lieber Herr Kollege Ledergerber

Ich verfolge mit Freude die drei Artikel [1-3] bzw. generell die Kommentare zum Thema Kosten im Gesundheitswesen.

\section{Sprechen wir im Gesundheitswesen die gleiche Sprache?}

Einmal mehr stelle ich fest, dass Gesundheitsökonom(inn)en (oder Analyst[inn]en von Statistiken) und Ärztinnen/Ärzte an der Front eine divergente Sprache sprechen. Ich erlaube mir einen Kommentar deshalb abzugeben, weil ich nach meinem Staatsexamen 1970 - im Lötschental und in Flamatt Praxisvertretungen gemacht habe, schliesslich Privatdozent und Leitender Arzt für Kinderchirurgie am Kinderspital Zürich wurde, eine eigene Station (für die Verbrennungsbehandlung bei Kindern) eröffnet und zehn Jahre geleitet habe und unterdessen seit 20 Jahren eine kinderchirurgische Tagesklinik mit meinen drei Kolleginnen betreibe. Sie sehen, dass ich damit vielleicht von der Arbeit an der Front ein wenig Ahnung habe und dass ich deshalb die «wissenschaftlichen» Analysen von Gesundheits- und Kostenstatistiken vorsichtig hinterfrage.

\section{Relevanz der Statistiken oder wie teuer darf es sein?}

Zur Relevanz ist effektiv zu sagen, dass die Verknüpfung einzelner Statistiken oft fehlt. Dass zum Beispiel von 100 Verkehrsopfern früher mehr starben als heute, kann ein Sieg der Vernunft, der Sicherheit der Fahrzeuge oder der Verkehrsberuhigungen sein. Einverstanden. Dies sagt aber nichts aus, ob die nun vermehrt Überlebenden auch "gesund» geworden sind. Liegen sie nicht vielleicht zwar überlebend, aber schwerstbehindert in einem Heim und belasten horribile dictu - unsere Sozialversicherungen?

Sie schreiben, dass die realen 59 Milliarden Franken unwiederbringliche Ausgaben seien. Sie verkennen dabei, dass dieses unwiederbringliche Geld nicht nur die bekanntermassen extremen Entlöhnungen von uns Ärzten oder unserer Krankenkassenkader sind, sondern eine erkleckliche Summe an Löhnen, rückfliessenden Steuern und Sozialabgaben sowie eine nicht unerheb- liche Wertschöpfung verschiedenster Wirtschaftszweige beinhalten. Im Medikamentenpreis ist und das sollten Gesundheitsökonomen auch einmal unserer Bevölkerung weismachen - nicht nur das Einkommen eines Herrn Vasella enthalten, sondern auch die Instandhaltung des Fabrikationsgebäudes und damit die Aufträge für das Bauhandwerk, der Lohn für den Transporteur der Medikamente in unsere Praxis und damit wieder der Umsatz seines Garagisten und des Tankstelleninhabers. So greift doch ein Rädchen ins andere, und die Couchepin'schen Sandkörner in diesem Getriebe bringen ausser einer Wiederwahl nur Friktionen und damit letztlich volkswirtschaftlich überhaupt nichts!

Dass die Prämien für einen grossen Teil unserer Bevölkerung extrem hoch sind, ist mir selbstverständlich auch bewusst. Wenn Sie die Gesundheitskosten (oder vielmehr den «Umsatz im Gesundheitsgewerbe») im Jahr 1969 in der Höhe von 4,6 Mrd. Franken einfach indexunkorrigiert mit den heutigen 59 Mrd. vergleichen, scheint mir dies ein wenig unprofessionell (auch wenn ich sicher nicht den gleichen ökonomischen Durchblick wie Sie besitze): 1966 betrug der Lebenshaltungskostenindex 100 Punkte, 2007 hingegen 337,1 Punkte ...

Die Betriebsausgaben der schweizerischen Krankenhäuser sind von rund 238000 Franken pro Bett im Jahre 1998 auf 361800 Franken im Jahre 2007 gestiegen, die Zahl der Beschäftigten in diesen Betrieben hat im gleichen Zeitraum von rund 97000 auf 130000 zugenommen. Die Werte von 1969 habe ich leider nicht zur Verfügung. Im gleichen Zeitraum hat der Anteil der über 65-Jährigen in der Schweizer Bevölkerung um 1,3\% zugenommen, dafür die Zahl der Spitalaustritte (bei über 70-Jährigen) von 196000 auf 366000 . Dies sind die statistischen Facts, die es zu vergleichen gilt, wenn wir etwas verändern wollen, nicht diejenigen irgendwo im Ausland!

Beispiel: Der UBS-Broschüre von 2003 ist bezüglich Kaufkraft und Lohnniveau zu entnehmen, dass für einen Big Mac in Zürich 14 Minuten, in Frankreich 19 und in Portugal 33 Minuten gearbeitet werden muss. Wie lassen sich schon nur auf diesem Niveau Vergleiche oder gar Rückschlüsse wirklich vornehmen? Sind Kaufkraft- 
paritäten im EU-Raum und medizinischer Outcome in die Vergleiche eingeflossen oder überhaupt messbar?

Es ist unter anderem nicht ein Problem des Gesundheitswesens und seiner Akteure, dass die Löhne für Medizinalpersonal gegenüber früher so stark angestiegen sind. Ich spüre dies Letztere sehr wohl, wenn wir für unsere 12 Mitarbeiterinnen pro Jahr eine Million Lohnkosten berappen, einen Betrag, der leicht bei einem nicht überrissenen Salär von 5500 bis 6000 Franken nachgerechnet werden kann! Andersherum - rechne: Wie viele Konsultationen sind nötig, damit allein die Lohnkosten unter Dach sind?

\section{Mehrkosten auch Mehrleistung?}

Bedeuten Mehrkosten auch Mehrleistung (s. Ihr 5. und 6. Abschnitt), ist eine ethische - und vielleicht sogar müssige Frage. Die medizinischen Leistungen haben sich verbessert - aber eben nicht parallel, sondern im Kostenbereich vor allem wegen der steigenden Fixkosten exponentiell für die entsprechende Leistung, die notabene allen Bewohnern dieses Landes zugänglich ist.

Ist die verlängerte Überlebenszeit von Karzinompatienten (oft als «Heilung» propagierte Zeit nach Diagnosestellung der Erkrankung) wirklich ein Gewinn, obwohl bekanntermassen die Kosten «für ein paar Monate mehr» extrem hoch sein können? Ist es sinnvoll, Frühgeborene von 500 Gramm am Leben zu erhalten, vielleicht mit ungewissem Ausgang? War es sinnvoll, mich im letzten Herbst anlässlich meines Herzversagens mit 64 Jahren noch zu reanimieren und zu defibrillieren, mich in der Intensivstation mit Hypothermie zu beatmen, mich anschliessend auf der Abteilung aufzupäppeln, um mich noch vier Wochen nach Gais zu schicken, damit ich nun noch einen Defibrillator implantiert bekomme? $\mathrm{Zu}$ meinen Staatsexamenszeiten war ein Herztod mit 64 «halt normal». Es wäre für die Krankenkasse, sprich meine solidarisch mitzahlenden Mitmenschen, natürlich viel billiger gewesen, nur die Ausstellung des Totenscheins durch den Notfallarzt übernehmen zu müssen anstelle meiner 100000-fränkigen Überlebensübung! (Und ich hätte diesen vielleicht unnötigen Artikel nicht geschrieben ...)

Es ist letztlich eine politisch-ethische Frage, die Sie und alle Spezialisten im Bereich Qualitätssicherung und Gesundheitsökonomie natürlich auch nicht beantworten können oder wollen, ob und warum und in welchem Alter bei uns Schweizern noch die an sich möglichen Massnahmen mit der entsprechenden Kostenfolge durchgeführt werden sollen, damit wir im internationalen Vergleich nicht mehr im Spitzenbereich liegen. Sarkastisch betrachtet heisst die Botschaft: Wer kommuniziert unseren Einwohnern, dass sie nun einmal nicht das ewige irdische Leben haben und dass mit der ersten Lebenssekunde der Tod mit inbegriffen ist?

\section{Vergleiche sind tot - es lebe der Vergleich?}

Ceterum censeo - die rein ökonomischen internationalen Vergleiche (und diese haben Sie trotz Ihrer Abrede letztlich kommentiert) sind zwar ganz witzig zum Lesen, helfen uns aber nur bedingt weiter. Dies heisst beileibe nicht, dass wir uns über unsere hiesige Entwicklung im Gesundheitswesen nicht Gedanken machen müssen!

Ich muss im konkreten Fall den Grundgedanken im Beitrag von Herrn Ledergerber mehr Gewicht als Ihrer Replik beimessen, auch wenn sie «statistisch = wissenschaftlich» vielleicht nicht dem Inhalt der 80-Euro-CD entsprechen. Vielleicht hat Herr Ledergerber «Äpfel mit Birnen» verglichen. Bedenken Sie aber, Äpfel aus Helsingborg schmecken auch anders als diejenigen aus Reggio di Calabria!

\section{Literatur}

1 Kocher G. Unser Gesundheitswesen im internationalen Vergleich. Schweiz Ärztezeitung. 2008;89(44):1911-6

2 Ledergerber J. Die Kosten unseres Gesundheitswesens. Schweiz Ärztezeitung. 2009;90(6):219-20.

3 Kocher G. Verzicht auf Vergleiche, Lob statt Kritik? Schweiz Ärztezeitung. 2009;90(6):221-2. 